\title{
Pharmacological and dietary agents for colorectal cancer chemoprevention: Effects on polyamine metabolism (Review)
}

\author{
MICHELE LINSALATA*, ANTONELLA ORLANDO* and FRANCESCO RUSSO* \\ Laboratory of Nutritional Pathophysiology, National Institute for Digestive Diseases, \\ I.R.C.C.S. 'S. de Bellis' Via Turi 27, Castellana Grotte (Ba), I-70013, Italy
}

Received May 26, 2014; Accepted July 4, 2014

DOI: 10.3892/ijo.2014.2597

\begin{abstract}
Chemoprevention is the long-term use of different chemical agents, both synthetic and natural, to prevent or delay the onset of disease. Since colorectal cancer has a significant environmental component, it is an ideal disease in which to evaluate the potential benefits of chemopreventive agents. The polyamines, spermine, spermidine and putrescine have been involved in almost all the steps of colorectal tumorigenesis. Consequently, polyamine biosynthesis and catabolism can be considered as promising targets for cancer chemoprevention. A variety of drug formulations have been tested for their efficacy in affecting polyamines in a strategy of colorectal cancer prevention. Different molecules, such as biosynthesis inhibitors and catabolism inducers, have been proposed alone or in combination with other drugs proved to diminish the colorectal cancer risk. Interestingly, also diet can play a role in cancer prevention by affecting polyamines. Several dietary components, such as probiotics or flavonoids, have been shown to affect the polyamine metabolic pathway in colorectal neoplastic tissue. On the other hand, the polyamines ingested with diet might contrast the above cited effects shown by both drugs and nutritional
\end{abstract}

Correspondence to: Dr Francesco Russo, Laboratory of Nutritional Pathophysiology, National Institute for Digestive Diseases, I.R.C.C.S. 'S. de Bellis', Via Turi 27, Castellana Grotte (Ba), I-70013, Italy

E-mail: francesco.russo@irccsdebellis.it

*Contributed equally

Abbreviations: CRC, colorectal cancer; ODC, ornithine decarboxylase; SAM-DC, S-adenosylmethionine decarboxylase; SSAT, spermidinespermine-N1-acetyl transferase; PAO, polyamine oxidase; AZ, antizyme; AZI, antizyme inhibitor; DFMO, difluoromethylornithine; APC, adenomatous polyposis coli; GI, gastrointestinal; NSAIDs, non-steroidal anti-inflammatory drugs; SNP, single nucleotide polymorphism; AOM, azoxymethane; L.GG, Lactobacillus rhamnosus strain GG; ERs, estrogen receptors; ACF, abberrant crypt foci; PPAR $\gamma$ peroxisome proliferator-activated receptor $\gamma$; EGCG, (-)-epigallocatechin-3-gallate; CRA, colorectal adenoma

Key words: colorectal cancer, chemoprevention, polyamines, difluoromethylornithine, flavonoids, probiotics, resveratrol factors. It is, therefore, fundamental to acquire more data also on these aspects in view of an innovative approach to colorectal oncology. This review summarizes data on the role of polyamine metabolism in neoplastic transformation of colorectal mucosa and as possible target for colorectal cancer chemoprevention. Attention will be focused on the influence of drugs and nutritional factors on polyamine metabolism, as well as the role played by dietary polyamines.

\section{Contents}

1. Introduction

2. Polyamine metabolism and colorectal cancer

3. Influence of drugs on polyamine metabolism for CRC chemoprevention

4. Influence of nutritional factors on polyamine metabolism for CRC chemoprevention

5. Influence of dietary polyamines on CRC chemoprevention

6. Conclusions

\section{Introduction}

Colorectal cancer (CRC) is one of the main causes of death in Western countries. Many colon cancer treatment options are available, including surgery, chemotherapy and radiation, but chemoprevention is a fundamental approach to reduce cancer risk. Chemoprevention is the long-term use of different chemical agents, both synthetic and natural, by healthy individuals to prevent or delay the onset of disease. Since CRC has a significant environmental component, it is an ideal disease in which to evaluate the potential benefits of chemopreventive agents. Different CRC chemoprevention strategies are under investigation. They include prevention of radical formation and DNA hypomethylation, prevention or suppression of mutations, inhibition of cell proliferation and induction of tumor cell differentiation (1).

Among the several biochemical alterations found in cancer cells, one of the most noticeable is a change in the intracellular polyamine content. Polyamines are polycationic compounds that play a key role in almost all the steps of colorectal tumorigenesis. In CRC tissue, the polyamine content as well as the activities of two important enzymes in their biosynthesis such as ornithine decarboxylase (ODC) 
and S-adenosylmethionine decarboxylase (SAM-DC), are increased 3-4 fold over that found in the equivalent normal colonic tissue. Besides, polyamines have been considered as possible markers of neoplastic proliferation in the colon. Noticeably, it is not only polyamine synthesis, but also their uptake that is enhanced in rapidly proliferating neoplastic cells in the colon. In contrast to all other cell systems in the body, colon cancer cells are exposed to high concentration of polyamines. These derive from the cells of the gut mucosa, which release their polyamine content into the lumen during the process of cell extrusion and death, but also from the gut bacteria and food. Therefore, also the introduction of polyamines by exogenous environment with diet may be of importance in a strategy of cancer prevention (2).

This review summarizes data on the role of polyamine metabolism in neoplastic transformation of colorectal mucosa and as possible target for CRC chemoprevention. Attention will be focused on the influence of drugs and nutritional factors on polyamine metabolism, as well as the role played by dietary polyamines.

\section{Polyamine metabolism and colorectal cancer}

Polyamines (putrescine, spermidine and spermine) are organic cations present in every living cell. They have pleiotropic effects on cell physiology and play a relevant role in cell proliferation and differentiation (3). These molecules are positively charged at the primary and secondary amino groups at physiological $\mathrm{pH}$. Thus, polyamines may act as ligands at multiple sites on DNA, RNA, proteins, phospholipids and nucleotide triphosphates (4). Accordingly, biological functions of polyamines are mainly involved in the regulation of gene expression by altering DNA structure and modulating signal transduction pathways $(5,6)$.

The polyamine functions and their metabolic pathway have been extensively studied $(7,8)$. Fig. 1 shows a schematic representation of the polyamine metabolic pathway. Briefly, biosynthesis is mediated by the key enzyme ODC which converts the amino acid ornithine into putrescine. This is then sequentially converted into spermidine and spermine, through the action of the enzyme SAM-DC and spermidine/spermine synthase. The central enzyme in the polyamine catabolic pathway is the spermidine-spermine-N1-acetyl transferase (SSAT), which adds acetyl groups to terminal amine groups in spermidine and spermine. These acetylated polyamines are then substrates for the enzyme polyamine oxidase (PAO) which retro-converts these acetylated derivatives into lower chain amines.

Intracellular levels of polyamines are tightly controlled and this occurs in addition to the multi-level control of synthesis and catabolism, also by uptake and efflux (9).

The protein ornithine decarboxylase antizyme (AZ) can effectively control polyamine levels not only by inactivating ODC and inducing its degradation, but also by increasing polyamine efflux and decreasing polyamine uptake. However, an antizyme inhibitor (AZI) has been characterized and the overexpression of this compound in certain forms of cancer has been reported (10).

Many biochemical alterations have been found in cancer cells, but one of the most consistent is a change in the intracel- lular polyamine content. Polyamine concentrations increase during carcinogenesis and an increase in ODC activity accompanies neoplastic transformation (11). As with other tumors, polyamine content of CRC is higher when compared to the adjacent mucosa and equivalent normal tissue $(12,13)$. The increase is due to the loss in polyamine homeostasis occurring during the dysregulation of cell proliferation (2). This is also proven by evidence of an upregulation of polyamine biosynthesis (14), a decrease in their catabolism $(15,16)$ and an increased uptake (17). Since polyamine metabolism is an integral component of the mechanism of the carcinogenesis in colorectal tissue, polyamine levels and ODC activity have been considered even as specific markers for neoplastic proliferation in the colon (18). Particularly, ODC has been shown to be critical in cell transformation and it has been suggested to be a proto-oncogene (19).

A possible role of polyamines in regulating oncogene expression and function through transcriptional and postranscriptional processes has also been suggested (20). On the contrary, two of the most commonly mutated genes in colon cancer, such as the adenomatous polyposis coli (APC) tumor suppressor gene and K-ras oncogene, have been shown to regulate the expression of several polyamine metabolic genes (e.g., ODC and SAT) (15,21). In this context, it has also been observed that polyamine biosynthesis is involved in human colorectal carcinogenesis in a manner that is K-rasdependent and p53-independent; besides, K-ras mutation and polyamine biosynthesis seem to be preferentially associated with polypoid tumors rather than flat colorectal tumors of the colon $(22,23)$. Given that polyamines and cancers seem to be tightly connected, modulations of the polyamine metabolic pathway such as polyamine uptake and efflux, have received much attention in cancer drug development for chemoprevention of human CRC (24).

\section{Influence of drugs on polyamine metabolism for CRC chemoprevention}

ODC, being the rate limiting enzyme in polyamine biosynthesis, has represented the first target in the polyamine pathway for cancer therapy. Several studies have focused on the use of difluoromethylornithine (DFMO), an ODC inhibitor, as chemopreventive and chemotherapeutic agent $(25,26)$. Although DFMO has been proven to inhibit the growth of tumor cells in vitro, it has not been so convincing in its anti-neoplastic properties, being cytostatic rather than cytotoxic, at least in vivo (27). Besides, prohibitively high doses of DFMO were usually required to inhibit malignant tumor growth in early chemotherapeutic trials $(28,29)$.

The lack of efficacy by DFMO against established tumors has been put in relation to the availability of extracellular polyamines, derived from the diet, the retroconversion pathway as well as the polyamine content supplied by the gastrointestinal (GI) microbiota (30). However, it has also been observed that very low, non-toxic doses of DFMO were able to inhibit stimulation of proliferation by various carcinogens and this evidence has led to investigation of DFMO as a cancer chemopreventive agent. Probably, the greatest potential for DFMO action in chemoprevention may be exerted against $\mathrm{CRC}$, where the decrease in ODC activity and polyamine 


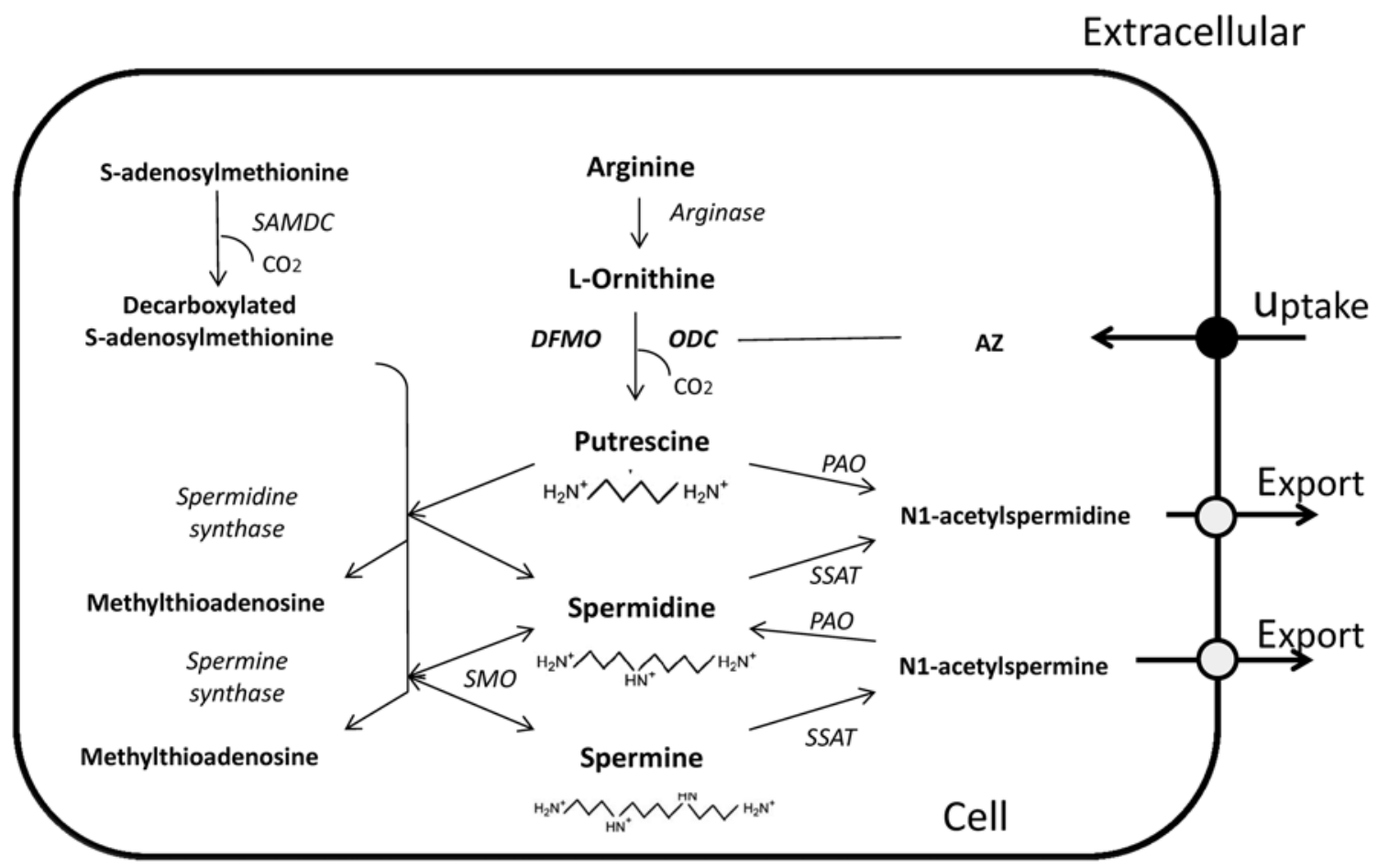

Figure 1. Polyamine metabolic pathway. ODC, ornithine decarboxylase; SAM-DC, S-adenosylmethionine decarboxylase; SSAT, spermidine-spermine-N1-acetyl transferase; PAO, polyamine oxidase; AZ, antizyme; DFMO, difluoromethylornithine; SMO, spermine oxidase.

content have been proven to limit significantly the formation of tumors (31).

A series of problem must be solved before conclusive evidence might be obtained from chemopreventive trials. Firstly, the putative chemopreventive agents have to be administered over long periods of time. Therefore, a major problem to be resolved concerns not only efficacy, but also the presence of minor toxicity and unpleasant side effects. Secondly, appropriate intermediate and endpoint biomarkers which allow the monitoring of the effectiveness of the drugs must be identified before the start of the research (32). In this connection, the early prevention clinical trials of DFMO have been conducted including pilot phase IIa and IIb studies aimed at evaluating the safety of treatment as well as the effective reduction in the colorectal tissue polyamine content (33).

It has been observed that an oral dose of $0.2 \mathrm{~g} / \mathrm{m}^{2} /$ day can be both effective in reducing colorectal mucosal polyamine contents and safe, given that no relevant signs of toxicity were observed in the treatment group, compared to the placebo group (34).

It is known that combining different molecules in a strategy of chemoprevention could offer the prospect of reduced toxicity by lowering doses of individual agents. Therefore, a variety of combined formulations in order to decrease intracellular polyamine levels have been proposed and studied for their efficacy in cancer prevention. As an example, one successful strategy is based on the administration of DFMO along with non-steroidal anti-inflammatory drugs (NSAIDs). As demonstrated by epidemiological reports, a regular use of NSAIDs is associated with a decreased risk for CRC (35), whereas experimental studies have shown that DFMO acts, at least additively, with a number of NSAIDs (36).
This drug combination proved to decrease polyamine synthesis by inhibiting ODC (action due to DFMO), while contemporary increasing catabolism and cellular export of polyamines by activating SAT (induced by NSAIDs) (37). This combination produced an additive reduction of colon carcinogenesis also in laboratory animals (38) and it was observed that sulindac and celecoxib are both potent stimulators of SAT and inhibitors of intestinal carcinogenesis in Apc Min/+ mouse (39).

More recently, a 3-year trial (40) strongly validated a significant effect of the combined administration of low doses of DFMO (500 mg/die) plus sulindac $(150 \mathrm{mg} / \mathrm{die})$ on the polyamine production in rectal mucosa. Although no relationship between changes in polyamine levels and response were observed, the baseline polyamine levels affected the DFMO plus sulindac effects for colorectal adenoma (CRA) prevention.

By combining DFMO with NSAIDs, or other proven chemopreventive agents, it is also expected that fewer side effects occur during treatment. In this connection, it has been reported that DFMO plus sulindac administration is safe and effective in chemoprevention of CRA in people with prior colon polyps (41). However, other phase III trials involving adenoma patients, showed that the same combination of DFMO plus sulindac is actually effective, but confers a modest risk of ototoxicity and the potential risk of cardiovascular toxicity (42).

The usual side-effect ototoxicity and differential treatment outcomes related to the DFMO plus sulindac treatment could be associated with the specific germline single nucleotide polymorphism (SNP) in the ODC-1 promoter region. SNP has been investigated as a marker for colorectal adenoma (43). In this connection, it has been observed that aspirin is able to decrease the risk of CRA recurrence especially in individuals homozygous for the ODC1 minor A allele compared with 
those with the major $\mathrm{G}$ allele (44). This has been confirmed and corroborated by other studies (45). These findings suggest that genetic features of the polyamine metabolism may be markers for both treatment benefit and toxicity.

Some data also indicate a potential link between obesity and polyamine inhibition in humans (46); besides, an association between obesity and risk of CRA has been reported (47). In spite of these observations, in a recent study it has been shown that obesity does not substantially modify the CRA risk reduction ascribed to DFMO plus sulindac versus placebo (48). This evidence strongly supports the need for chemopreventive clinical trials to refine the risk:benefit as well as the risk:risk profiles of the putative chemopreventive agents.

Based on the above, it is evident that the colorectal chemoprevention strategy with DFMO plus NSAIDs provides important proofs of principle that targeting polyamine metabolism can really be an effective strategy for reducing those risk factors closely associated with the development of colon cancer in humans.

Apart from ODC, the other polyamine biosynthesis enzymes, SAM-DC and spermidine/spermine synthase, have also received considerable attention as target for polyamine level reduction. Most SAM-DC inhibitors rapidly depleted spermidine and spermine concentrations in the cells, but their effects disappeared when in vivo models were tested. Probably, this happened because the accumulation of putrescine might compensate for spermidine in cells. Inhibitors of spermidine/spermine synthase have not been shown to be efficacious in either in vitro or in vivo system (49). PAO inhibitors have also been developed, since this enzyme converts spermine to spermidine, in order to overcome cell growth inhibition by polyamine depletion. These inhibitors are potent killers of cancer cells in vitro and showed promise when used with DFMO in carcinogenesis model in vivo $(25,50)$.

As above mentioned, in addition to inhibition of polyamine biosynthesis, induction of polyamine catabolism has also been a major pharmacological goal. The enzyme SSAT can be induced by a variety of compounds like NSAIDs and polyamine analogues (51). These latter molecules need a special mention, due to their pleiotropic effects on cancer cells. Polyamine analogues are able to reduce the cell polyamine content by both upregulating catabolism (inducing SSAT), decreasing biosynthesis by negative feedback inhibition and by competing with exogenous polyamines for uptake. Besides, polyamine analogues can inhibit cell growth by acting like endogenous polyamines and bind to intracellular polyamine binding sites, thus rendering them 'non-functional' (52). In vitro studies have clearly demonstrated a role for polyamine analogues as multi-site inhibitors of the polyamine pathway, although the efficacy of each type of analogue in cancer chemoprevention and therapy remains to be fully characterized in vivo (53).

Other aspects of the polyamine metabolism pathway including polyamine uptake and efflux have also been targeted for cancer drug development. Uptake inhibitors have shown promise in vitro and have increased the efficacy of DFMO as an anticancer agent $(17,54)$.

Aside from specific inhibitor/inducer drugs for the polyamine pathway and transport, also several nutritional components thought to be useful in colon chemoprevention have been shown to affect the polyamine metabolic pathway as well as to impair the tissue polyamine content in colorectal neoplastic tissue.

\section{Influence of nutritional factors on polyamine metabolism for CRC chemoprevention}

The GI tract is constantly connected with the external environment, therefore all the possible modifications in daily diet might significantly modify the exposure to different carcinogenetic factors. In this framework, the identification of those components in diet that may display at different degree some antitumor activity as well as the understanding of their mechanisms of action, may lead to significant advances in human cancer prevention. Several diet components have been demonstrated as having some anti-neoplastic activity. From the early 1970s, many studies following different designs (e.g., correlation studies, case-control, cohort studies) have hypothesized that dietary fibers may show protective effects against diverse neoplasms, including GI neoplasms (55). Their positive action in the GI environment has been essentially put in relation with the bacterial strains resident in the GI lumen (56).

The intestinal microbiota have been considered as a potential target for an active anticancer strategy. In view of its fundamental role in human health, manipulation of the intestinal microbiota by microrganisms shown to positively affect the GI tract (e.g., probiotics) or other compounds found in plants, has been considered as a logical approach to prevent or inhibit the neoplastic transformation of GI mucosa.

Probiotics are defined as 'live microorganisms which, when administered in adequate amounts, confer a health benefit on the host' (57). Together with these positive bacterial strains, other substances, mainly found in plants have been deeply investigated for their chemopreventive and chemotherapeutic properties against human cancers. These substances include flavonoids such as genistein (found in soy), quercetin (onions), apigenin (celery, parsley), green tea (polyphenols), etc. On these bases this chapter tries to review data on those nutritional components useful for CRC chemoprevention in relation to their potentiality in affecting the polyamine metabolism.

Probiotics. At present, the most commonly used microorganisms for manipulating the GI environment belong to Lactobacillus and Bifidobacterium genera (58). These two genera contain several species and strains of which many are being used as probiotic strains and most of them are categorized as lactic acid-producing bacteria (59). Usually, probiotics are consumed in the form of yogurt, fermented milks or other fermented foods, even if more recent studies have proposed their administration by using vegetables (e.g., artichokes, olives) as carriers (60-62). Their end products are mainly organic acids (lactic and acetic acids) that tend to lower the $\mathrm{pH}$ of the intestinal content, creating a less favorable environment for harmful bacteria.

Several health-promoting effects have been attributed to the probiotic lactic acid bacteria, but the most interesting and controversial is their anticancer activity against different neoplasms, including CRC (63).

These bacteria have been shown to possess antimutagenic and anticarcinogenic properties and data from epidemio- 
Table I. Evidence for antineoplastic mechanisms of action of probiotics.

a. Reduction in the activity of fecal enzymes (betaglucuronidase, azoreductase, nitroreductase and 7 -alphadehydrogenase) considered as having a role in colon cancer.

b. Reduction in the incidence of chemically induced tumors in rats.

c. In vitro prevention of damage to DNA in colon cancer cell lines.

d. In vitro binding of mutagens by different components of probiotic bacteria.

e. Improvement of immune system defense.

f. Reduction in the polyamine content in colon cancer cell lines and in laboratory animals.

logical and experimental studies clearly indicate that ingestion of lactobacilli and bifidobacteria, or their fermented dairy products, reduce the risk of certain types of cancer and inhibit tumor growth $(64,65)$. The postulated mechanisms involve alterations of luminal $\mathrm{pH}$ in the gut, production of antimicrobial compounds, competition for pathogen binding and receptor sites, competition for available nutrients and growth factors, stimulation of immunomodulatory cells, and production of lactase (66). Table I reports some of the evidence for antineoplastic actions by probiotics.

Some studies have emphasized a relationship between polyamine biosynthesis and probiotic action in carcinogenesis and tumor growth. In studies performed in mice (67), the administration of Bifidobacterum longum cultures significantly suppressed the rate of cellular proliferation, the expression and activity of ODC, as well as mutated ras-p21 in a manner strongly correlating with inhibition of tumor induction by azoxymethane (AOM). Lactobacillus brevis strain showed pro-apoptotic effects in Jurkat cells and it was hypothesized that this ability in inducing apoptosis could be associated with polyamine synthesis (68). Besides, a peculiar Lactobacillus brevis strain (CD2 strain) demonstrated anti-proliferative biochemical features, essentially related to the activity of arginine deiminase (69). This enzyme is able to catalyse the catabolism of arginine and to affect the biosynthesis of polyamines (70). Di Marzio et al (68) advanced the hypothesis of an involvement of arginine deiminase in a study performed on the human $\mathrm{T}$ leukemia Jurkat cell line. The authors demonstrated that lyophilized and sonicated preparations of L. brevis (CD2) were able not only to cause arginine-dependent polyamine synthesis inhibition, but also to induce consequently a relevant apoptotic effect.

Previously, Orlando et al (71) performed a study aimed at investigating the effects of increasing concentrations of Lactobacillus rhamnosus strain GG (L.GG) homogenate on cell growth and proliferation in neoplasms originating from different GI tracts, such as gastric HGC-27 and colonic DLD-1 cells, focusing attention in their polyamine profile and biosynthesis. Additionally, in order to verify which bacterial fraction was involved in the anti-proliferative effects, the cytoplasm and cell wall extracts were tested separately. Both cell lines proved to be sensitive to the growth inhibition by the highest concentrations of bacterial homogenate with a significant reduction in their polyamine concentrations. Interestingly, either HGC-27 or DLD-1 cells were resistant to the bacterial cell wall fractions, whereas increasing cytoplasm fraction concentrations induced an evident anti-proliferative effect. These data suggested that cytoplasm extracts could be responsible for $L . G G$ action on proliferation of these two cell lines from gastric and colonic neoplasms. Another probiotic, Saccharomyces boulardii (S. boulardii) usually prescribed in a lyophilized form, demonstrated not only to act as a carrier able to release different active metabolic compounds such as enzymes and trophic factors during its intestinal transit, but also to secrete its polyamine content (mainly spermine and spermidine), thus directly affecting gene expression and protein synthesis (72).

Also the effects of a probiotic mixture of 8 different bacterial strains (VSL\#3) on polyamine biosynthesis, Ki-67 levels and apoptosis in the normal colon of rats have been evaluated (73). It has been postulated that probiotic mixtures may have a higher efficacy than single strains due to an additive or even synergistic effects when put together with other probiotic strains. The combined use of these probiotic strains (S. thermophilus, B. breve, B. longum, B. infantis, L. acidophilus, L. plantarum, L. casei, L. bulgaricus) caused a significant decrease in colonic polyamine levels, ODC activity and Ki-67 compared to controls along with a significant increase in the apoptotic index. In this framework, the results of this study suggested that probiotics could also reduce proliferation rates in a normal condition not affected by hyperproliferative or neoplastic growth.

In humans, the effects on fecal probiotic metabolites (polyamines, lactate and acetate) and mutagenicity following administration of a yogurt containing Bifidobacterium lactis LKM512 were investigated in healthy adults (74). Consumption of LKM512 yogurt increased fecal spermidine levels, but not fecal lactate and acetate contents and significantly reduced the mutagenicity level to $79.2 \%$. These results allowed the authors to hypothesize that increased gut spermidine level by LKM512 yogurt was responsible for the reduction of mutagenicity in the human. In this connection, the link between cell proliferation, polyamines and probiotics could be regulated not only by the peculiar metabolic features of the microbial strains, but also by the period of administration or the proliferative behavior of different segments of GI mucosa. On this basis, and in view of the potential offered by probiotics in affecting the proliferative activity of GI mucosa, the possible implications in humans still deserve further deeper investigations.

Flavonoids. Other substances with postulated anti-neoplastic properties naturally present in foods are flavonoids. This group include phytoestrogens, plant compounds with estrogen-like activities. The main varieties are isoflavones such as genistein and daidzein found predominantly in soy products (75), and lignans found in whole grains, vegetables, fruits and flax seed (76).

Epidemiologic reports suggest that phytoestrogens contained in soy are causally related to protection against hormone-dependent cancers, probably by competing with estradiol for estrogen receptors (77) and data from literature 
provide evidence of an association between high consumption of phytoestrogen-rich foods and a low incidence of breast and prostate cancer within populations of Asian countries (78). There are also data regarding these substances and colonic neoplastic transformation with a significant reduction in CRC risk (79). The evidence is important, because dietary intake is modifiable. Therefore, identifying dietary phytoestrogens with antitumor activity and investigating their mechanisms of action may lead to significant advances in the prevention of human cancer. Also cell-culture studies and animal experiments have shown that flavonoids are tumor-inhibitory for CRC (80). It has been observed that ODC activity and polyamine concentrations were significantly lower in the mammary epithelium of rats treated with soy protein than in controls (81). Previous studies clearly demonstrated that, although the GI tract is not a classical sex hormonal target, sex steroid hormones can affect cell proliferation and turnover, playing an important role also in the neoplastic transformation in the colon $(82,83)$. This evidence suggests that estrogens as well as phytoestrogens introduced with diet may exert a protective role against CRC also in humans regardless of gender (84).

Among flavonoids, phytoestrogen genistein has drawn attention in recent years due to a variety of biological activities that may account for its cancer-preventive effects. In vitro experiments demonstrated that genistein influences proliferation, differentiation and apoptosis in different tumor cell types (85). The anticancer effects of genistein involve inhibition of angiogenesis, topoisomerase, tyrosine kinase activity and antioxidant processes (86).

Genistein possesses noticeable structural similarity with estrogens and effects resembling estrogenicity. This phytoestrogen is able to bind both the estrogen receptors (ERs) $\alpha$ and $\beta$. As cited above, both in vivo and in vitro studies clearly established that estrogens can exert an inhibitory effect on GI cell proliferation by interacting with growth factors, apoptotic processes and polyamine metabolism $(82,83)$. In this connection, it has been demonstrated that genistein inhibited cell proliferation in ER-positive (MCF-7) and ER-negative (MDA-MB-231) human breast carcinoma cell lines. In addition, ODC activity was reduced to $53.8 \%$ of the control after 6-h treatment with $50 \mathrm{mM}$ genistein in MCF-7 breast cancer cells (87).

Research by our group investigated the effects on the polyamine biosynthesis and cell growth following administration of increasing concentrations of genistein (from $0.01 \mu \mathrm{M}$ up to $100 \mu \mathrm{M}$ ) in the DLD-1 human colon cancer cells (88). This cell line has the peculiarity of being ER-positive. Starting from $1 \mu \mathrm{M}$, genistein administration reduced significantly ODC activity compared to untreated cells. Regarding the polyamine profile, the administration of increasing concentrations of genistein (namely, from 0.01 to $100 \mu \mathrm{M}$ ) decreased the single and total polyamine contents. Besides, the polyamine content was inversely correlated to genistein concentrations. When DLD-1 cell line was depleted by culture of the estrogenic content and exposed to increasing genistein concentrations, it also showed an evident pro-apoptotic (increase in Bax mRNA expression) and anti-proliferative action. It is conceivable that mechanisms by which genistein affects growth of DLD-1 cells may include either induction of apoptosis or a decrease in cell proliferation rate by involving a lessening in ODC activity and the polyamine levels. Of note, these results were obtained in vitro using a wide range of genistein concentrations with values falling within the physiological blood levels in human, further supporting indications for a diet rich in this isoflavone in terms of CRC prevention.

The modulating effects of dietary feeding of two flavonoids, diosmin and hesperidin were investigated in male F344 rats during the initiation and post-initiation phases on colon carcinogenesis initiated with AOM (89). In that study, the incidence and number of neoplasms in the gut of F344 rats together with, or followed by, a diet containing diosmin or hesperidin were significantly smaller than those of rats receiving AOM alone. Besides, administration of diosmin and hesperidin, alone or in combination, was able to significantly inhibit the development of aberrant crypt foci, the ODC activity in colonic mucosa as well as to reduce polyamine levels in the blood. As a consequence, it is conceivable that the significant anticancer properties shown by these flavonoids may be partly ascribed to their antiproliferative effects through the suppression of ODC activity and polyamine biosynthesis. In this framework, the polyamine content in both blood and tissue, may be one of the intermediate biomarkers.

The dietary flavonoid apigenin found in many fruits and vegetables, with parsley, celery and chamomile tea showing the highest amount, has been demonstrated to significantly inhibit at 10 and $30 \mu \mathrm{M}$ the ODC activity of Caco-2 cells. Besides, ODC activity in the colon mucosa of $\mathrm{CF}-1$ mice was reduced with $0.1 \%$ dietary apigenin by $42 \%$ compared with the control. Aberrant crypt foci (ACF) formation was also reduced by $50 \%$ with $0.1 \%$ dietary apigenin in AOM-induced CF-1 mice (90).

Another study (91) demonstrated that quercetin, found in fruits and vegetables such as citrus fruits, apples, onions, parsley, sage, tea and red wine, can affect proliferation, differentiation and apoptosis of DLD-1 cells by both decreasing polyamine biosynthesis and inducing apoptosis. At concentrations $\geq 50 \mu \mathrm{M}$, quercetin significantly reduced ODC activity, putrescine and spermidine levels compared to controls cells. Higher quercetin concentrations $(\geq 70 \mu \mathrm{M})$ caused a significant reduction in the conversion of MTT tetrazolium salt and $\left[{ }^{3} \mathrm{H}\right]$-thymidine incorporation. The same concentrations were needed to induce the apoptosis.

The apoptotic effects of apple procyanidins, oligomeric compounds formed from catechin and epicatechin molecules, were also established to involve the inhibition of polyamine catabolism (92). Procyanidins caused an activation of the intrinsic apoptotic pathway through enhanced polyamine catabolism and mitochondrial membrane depolarization. Besides, they caused a profound intracellular depletion of polyamines in SW620 cells. Apple procyanidins diminished the activities of ODC and SAM-DC, key enzymes of polyamine biosynthesis; the latter induced spermidine/spermine N1-acetyltransferase, which, in turn, started polyamines retroconversions. As a consequence of the enzymatic changes, polyamine concentrations diminished, and N(1)-acetyl-polyamines accumulated in SW620 cells. The observation that apple procyanidins enhance polyamine catabolism and reduce polyamine biosynthesis activity similar to known inducers of SSAT, without sharing their toxicity, let the author hypothesized that apple procyanidins could be useful for chemopreventive and therapeutic interventions. 
More recently (93), a metabolomic study on the anti-proliferative effect of dietary polyphenols on human colon cancer cells was conducted by using different methodological approaches. CE, RP/UPLC and HILIC/UPLC all coupled to TOF MS were combined to achieve a global metabolomic examination of the effect of dietary polyphenols on HT-29 colon cancer cells. Diverse metabolites, showing different expression after the treatment with polyphenols, were identified in colon cancer cells. Significant alterations in polyamine content along with changes in glutathione metabolism with more reduced glutathione/oxidized glutathione (GSH/GSSG) ratio were observed after the treatment with polyphenols in polyphenols-treated cells. These results from metabolomics further support the chemopreventive effect of the tested dietary polyphenols on colon cancer.

In conclusion, a plethora of plants and plant products contains metabolically active substances with anti-neoplastic properties, thus the possibility to regard flavonoids as representative phytochemical functional foods has made them attractive for in vivo studies on cancer risk.

Resveratrol. Resveratrol is a polyphenol classified as a phytoalexin, contained in grapes, wine and peanuts. Resveratrol seems to be, at least in part, responsible for the positive effects of a moderate red wine consumption on the development of cardiovascular diseases (94). Additionally, it has been reported that the resveratrol and its analogues have a potent chemopreventive effect in multiple carcinogenesis model in either in vivo or in vitro studies $(95,96)$. It is likely that the anticancer and the chemopreventive activities of resveratrol and its analogues could also be explained by the influence on polyamine metabolism. Different evidence was derived from in vitro studies. Schneider et al (97) reported that Caco-2 colorectal adenocarcinoma cells administered with $25 \mu \mathrm{mol} / 1$ resveratrol accumulated at the $\mathrm{S} / \mathrm{G} 2$ phase transition of the cell cycle, causing a $70 \%$ growth inhibition. Resveratrol produced a significant decrease of ODC activity with a concomitant reduction of the intracellular putrescine and spermidine content. Moreover, 24-h treatment with the resveratrol analogue cis-3,5,4' trimethoxystilbene decreased ODC and SAM-DC activities at a concentration of $0.3 \mu \mathrm{mol} / 1$ associated with a reduction of the putrescine content (98).

In Caco-2 cells, resveratrol was also proved to induce modification of polyamine metabolism (99) by inhibiting ODC activity and mRNA levels. c-Myc protein that controls the ODC promoter diminished by resveratrol treatment, demonstrating that decreased expression of the ODC gene is responsible for the inhibition of ODC activity. SAM-DC was also inhibited when high concentrations $(>50 \mu \mathrm{mol} / \mathrm{l})$ were used. In addition, resveratrol upregulated SSAT activity, inducing polyamine degradation. The SSAT gene is a target for the transcription factor peroxisome proliferator-activated receptor $\gamma(\operatorname{PPAR} \gamma)$ and Ulrich et al (100) postulated that p38MAPK and transcription factor PPAR $\gamma$ can be considered as molecular targets of resveratrol in the regulation of cell proliferation and SSAT activity, respectively.

In conclusion, the potential inhibitory effect of resveratrol on polyamine metabolism in carcinoma cells could be mediated by two different pathways: inhibition of polyamine synthesis and increased polyamine catabolism (101).
Green tea and (-)-epigallocatechin-3-gallate (EGCG). Green tea, widely consumed in Far East countries, contains polyphenolic compounds which account for $30 \%$ of the dry weight of the leaves. Most of the polyphenols are flavanols, and EGCG is the most abundant and representative for its putative antineoplastic effect. Green tea plays an important role in reducing cancer risk and in delaying cancer outbreak and recurrence (102) and epidemiological studies have revealed that the incidence of stomach and prostate cancers are low among populations that introduce regularly green tea in their diet. Various experimental studies performed in vivo and in vitro have confirmed the anticancer effects by green tea and/or EGCG (103).

It has been shown that green tea and its active components interfere with signal transduction pathways and mRNA expression in human colon cancer cells (104) and data are available on the possible relationship between green tea and/or EGCG and polyamine metabolism (105). Earlier studies demonstrated that in rodents with skin tumor induced by carcinogens, the application of EGCG was able to prevent the neoplasm onset and contemporary inhibited the ODC expression (106). These results suggest that EGCG may be an effective chemopreventive agent in individuals with early, pre-neoplastic stages of cancer.

The ODC/Ras double transgenic mouse model that develops spontaneous skin tumors due to overexpression of ODC and a v-Ha-ras transgene was used as a model to test the administration of EGCG in the drinking water. EGCG significantly decreased both tumor number and total tumor burden compared with untreated ODC/Ras mice without decreasing the elevated polyamine levels present in the ODC/Ras mice. EGCG selectively decreased both proliferation and survival of primary cultures of ODC overexpressing transgenic keratinocytes, but not keratinocytes from normal littermates nor ras-infected keratinocytes. This decreased survival was due to EGCG-induced apoptosis and not terminal differentiation. Moreover, in skin from EGCG-treated ODC transgenic mice, caspase 3 was detected only in epidermal cells that possess very high levels of ODC protein (107).

Other studies have demonstrated that ECGC inhibited MAPK activity as well as the syntheses of Jun and Fos, in this way acting in a manner similar to DFMO (108). Moreover, it has been observed that feeding mice with green tea polyphenols for 7 days abolished the typical over-production of ODC in prostate cancers, also in this case showing effects similar to those by DFMO (109).

On these bases, it could be hypothesized that both EGCG and DFMO may share some anticancer and chemopreventive activities by inhibiting ODC with the undoubted advantage that, unlike DFMO, green tea and/or EGCG are natural products, which can be consumed at large quantities without any harmful side-effects (106).

\section{Influence of dietary polyamines on CRC chemoprevention}

In addition to endogenous polyamines, dietary polyamines and their metabolites by intestinal microorganisms have been shown to be major determinants of the total body polyamine pool. The colonic lumen contains polyamines from both the diet or exported by enteric bacteria and these polyamines are transported via mechanisms not yet well described on the apical cell surface (110). As far as diet is concerned, poly- 
amine content is high in several food products including fruits, cheeses and meat. Again, high concentrations of putrescine can be found in common diets, particularly in orange and grapefruit juice (111).

The early studies have shown that intestinal and dietary polyamines can enhance colonic tumorigenesis, and can minimize the effects of ODC inhibitors (112). In tumor-bearing animal models, it has been demonstrated that a polyamine deficient diet significantly enhances the antitumoral effect of DFMO plus neomycin (113). Overall, the use of polyamine transport inhibitors, alone or in combination with DFMO, provides a method to target cancers with high polyamine requirements (114).

It has been observed that the primary effect of dietary putrescine was to increase tumor grade; besides the effectiveness of sulindac to suppress intestinal carcinogenesis was partially abrogated by dietary putrescine in a murine FAP model (115).

Polyamine metabolism is also dependent on levels of the precursor amino acids arginine and ornithine. In a study by Yerushalmi et al (116), Apc Min/+ mice were fed with arginine concentrations corresponding to the higher range of arginine consumed by humans eating a western style diet. Authors reported that large amounts of arginine in diet increase colonic polyamine levels as well as carcinogenesis. This finding strongly supports the concept that dietary arginine could be a risk factor for colon carcinogenesis also in humans $(117,118)$. In this connection, it has been found that patients with a family history of CRC and reporting meat consumption in the highest quartile had a statistically significantly decreased overall survival and increased risk of death, compared with those in the lower quartiles (119).

Recently, it has also been demonstrated that an association between high dietary polyamine intake and CRA risk in humans can be modified by sex and ODC genotype. Particularly for women, the risk of CRC development showed a positive trend with increasing quartiles of polyamine intake, and participants with higher polyamine intakes and the ODC GG genotype had significantly higher odds of CRC compared with those subjects showing the same genotype but lower polyamine intake (120). Therefore, a diet low in polyamines could represent an adjunctive strategy to therapeutic prevention using polyamine-inhibitory agents.

Raj et al (121) have observed a significant interaction between treatment with DFMO plus sulindac and dietary polyamine intake on the risk of recurrent adenomas in a CRA prevention phase III clinical trial. Patients in the highest quartile of dietary polyamine intake have been found to have no significant metachronous adenoma risk reduction after treatment with DFMO plus sulindac, in contrast to a significant $81 \%$ risk reduction observed for patients in the lowest quartile of dietary polyamine intake.

Collectively, the above evidence suggests that dietary polyamines may be involved in human colon carcinogenesis; therefore, approaches to limit dietary polyamines may represent additional strategies for CRC prevention. In this context, special diets low in polyamines have already been developed for other type of cancers and it has been observed that the polyamine-lowering regimen is associated with improved pain control and survival characteristics (122). However, due to the abundance of polyamines in the food supply and the strong preclinical data that relate polyamine exposure to tumor growth, these findings still support the need for additional investigation of dietary polyamines in human health.

\section{Conclusions}

Polyamines and their enzymes are strongly related to neoplastic proliferation in the GI tract. Therefore, all the strategies of effective chemotherapeutic and chemopreventive interventions targeting polyamines, will certainly require a combinatorial approach directed towards all the multiple features shown by their metabolic pathway.

Several agents in diet, thought to be useful in CRC chemoprevention, have been shown to affect the polyamine metabolic pathways. A combined chemopreventive intervention using protocols based on the use of these agents, along with polyamine inhibitors and/or inducers, would enhance their properties representing a suitable alternative option for the management of CRC patients. Moreover, also dietary polyamines may be involved in human colon carcinogenesis, conditioning the effects of drugs and nutritional components. It is, therefore, fundamental to acquire more data on this aspect that could represent an innovative and interesting approach to colorectal oncology. Future studies will determine whether strategies targeting the polyamine pathways will contribute to prevention of colon cancer.

\section{References}

1. Surh YJ: Cancer chemoprevention with dietary phytochemicals. Nat Rev Cancer 3: 768-780, 2003.

2. Casero RA Jr and Marton LJ: Targeting polyamine metabolism and function in cancer and other hyperproliferative diseases. Nat Rev Drug Discov 6: 373-390, 2007.

3. Ramani D, De Bandt JP and Cynober L: Aliphatic polyamines in physiology and diseases. Clin Nutr 33: 14-22, 2014.

4. Thomas T and Thomas TJ: Polyamines in cell growth and cell death: molecular mechanisms and therapeutic applications. Cell Mol Life Sci 58: 244-258, 2001.

5. Rothenburg S, Koch-Nolte F, Rich A and Haag F: A polymorphic dinucleotide repeat in the rat nucleolin gene forms Z-DNA and inhibits promoter activity. Proc Natl Acad Sci USA 98: 8985-8990, 2001.

6. Iacomino G, Picariello G and D'Agostino L: DNA and nuclear aggregates of polyamines. Biochim Biophys Acta 1823: $1745-1755,2012$.

7. Igarashi $\mathrm{K}$ and Kashiwagi K: Modulation of cellular function by polyamines. Int J Biochem Cell Biol 42: 39-51, 2010.

8. Pegg AE and Casero RA Jr: Current status of the polyamine research field. Methods Mol Biol 720: 3-35, 2011.

9. Pegg AE: Mammalian polyamine metabolism and function. IUBMB Life 61: 880-894, 2009.

10. Coffino P: Regulation of cellular polyamines by antizyme. Nat Rev Mol Cell Biol 2: 188-194, 2001.

11. Bachrach U: Polyamines and cancer: minireview article. Amino Acids 26: 307-309, 2004.

12. Linsalata M, Caruso MG, Leo S, Guerra V, D'Attoma B and Di Leo A: Prognostic value of tissue polyamine levels in human colorectal carcinoma. Anticancer Res 22: 2465-2469, 2002.

13. Linsalata M, Giannini R, Notarnicola $M$ and Cavallini A: Peroxisome proliferator-activated receptor gamma and spermidine/spermine N1-acetyltransferase gene expressions are significantly correlated in human colorectal cancer. BMC Cancer 6: 191, 2006.

14. Shantz LM and Levin VA: Regulation of ornithine decarboxylase during oncogenic transformation: mechanisms and therapeutic potential. Amino Acids 33: 213-223, 2007.

15. Erdman SH, Ignatenko NA, Powell MB, et al: APC-dependent changes in expression of genes influencing polyamine metabolism, and consequences for gastrointestinal carcinogenesis, in the Min mouse. Carcinogenesis 20: 1709-1713, 1999. 
16. Casero RA and Pegg AE: Polyamine catabolism and disease. Biochem J 421: 323-338, 2009.

17. Palmer AJ and Wallace HM: The polyamine transport system as a target for anticancer drug development. Amino Acids 38: 415-422, 2010

18. Thompson PA and Gerner EW: Current concepts in colorectal cancer prevention. Expert Rev Gastroenterol Hepatol 3: 369-382, 2009.

19. Pegg AE: Regulation of ornithine decarboxylase. J Biol Chem 281: 14529-14532, 2006.

20. Xiao L and Wang J-Y: Posttranscriptional regulation of gene expression in epithelial cells by polyamines. Methods Mol Biol 720: 67-79, 2011.

21. Ignatenko NA, Babbar N, Mehta D, Casero RA Jr and Gerner EW: Suppression of polyamine catabolism by activated Ki-ras in human colon cancer cells. Mol Carcinog 39: 91-102, 2004.

22. Linsalata M, Notarnicola M, Caruso MG, Di Leo A, Guerra V and Russo F: Polyamine biosynthesis in relation to K-ras and p-53 mutations in colorectal carcinoma. Scand J Gastroenterol 39: 470-477, 2004.

23. Notarnicola M, Linsalata M, Caruso MG, et al: Genetic and biochemical changes in colorectal carcinoma in relation to morphologic characteristics. Oncol Rep 10: 1987-1991, 2003.

24. Babbar N and Gerner EW: Targeting polyamines and inflammation for cancer prevention. Recent Results Cancer Res 188: 49-64, 2011.

25. Seiler N: Thirty years of polyamine-related approaches to cancer therapy. Retrospect and prospect. Part 1. Selective enzyme inhibitors. Curr Drug Targets 4: 537-564, 2003.

26. Laukaitis CM and Gerner EW: DFMO: targeted risk reduction therapy for colorectal neoplasia. Best Pract Res Clin Gastroenterol 25: 495-506, 2011.

27. Gerner EW and Meyskens FL Jr: Polyamines and cancer: old molecules, new understanding. Nat Rev Cancer 4: 781-792, 2004.

28. Love RR, Carbone PP, Verma AK, et al: Randomized phase I chemoprevention dose-seeking study of alpha-difluoromethylornithine. J Natl Cancer Inst 85: 732-737, 1993.

29. Levin VA, Uhm JH, Jaeckle KA, et al: Phase III randomized study of postradiotherapy chemotherapy with alpha-difluoromethylornithine-procarbazine, N-(2chloroethyl)-N'-cyclohexyl-N-nitrosurea, vincristine (DFMO-PCV) versus PCV for glioblastoma multiforme. Clin Cancer Res 6: 3878-3884, 2000.

30. Leveque J, Burtin F, Catros-Quemener V, Havouis R and Moulinoux JP: The gastrointestinal polyamine source depletion enhances DFMO induced polyamine depletion in MCF-7 human breast cancer cells in vivo. Anticancer Res 18: 2663-2668, 1998.

31. Meyskens FL Jr and Gerner EW: Development of difluoromethylornithine (DFMO) as a chemoprevention agent. Clin Cancer Res 5: 945-951, 1999.

32. Gerner EW: Cancer chemoprevention locks onto a new polyamine metabolic target. Cancer Prev Res (Phila) 3: 125-127, 2010.

33. Love RR, Jacoby R, Newton MA, et al: A randomized, placebocontrolled trial of low-dose alpha-difluoromethylornithine in individuals at risk for colorectal cancer. Cancer Epidemiol Biomarkers Prev 7: 989-992, 1998.

34. Meyskens FL Jr, Gerner EW, Emerson S, et al: Effect of alpha-difluoromethylornithine on rectal mucosal levels of polyamines in a randomized, double-blinded trial for colon cancer prevention. J Natl Cancer Inst 90: 1212-1218, 1998.

35. Flossmann E and Rothwell PM: Effect of aspirin on long-term risk of colorectal cancer: consistent evidence from randomised and observational studies. Lancet 369: 1603-1613, 2007.

36. Lawson KR, Ignatenko NA, Piazza GA, Cui H and Gerner EW: Influence of K-ras activation on the survival responses of Caco-2 cells to the chemopreventive agents sulindac and difluoromethylornithine. Cancer Epidemiol Biomarkers Prev 9: 1155-1162, 2000.

37. Gerner EW and Meyskens FL Jr: Combination chemoprevention for colon cancer targeting polyamine synthesis and inflammation. Clin Cancer Res 15: 758-761, 2009.

38. Jacoby RF, Cole CE, Tutsch K, et al: Chemopreventive efficacy of combined piroxicam and difluoromethylornithine treatment of Apc mutant Min mouse adenomas, and selective toxicity against Apc mutant embryos. Cancer Res 60: 1864-1870, 2000

39. Ignatenko NA, Besselsen DG, Stringer DE, Blohm-Mangone KA, Cui $\mathrm{H}$ and Gerner EW: Combination chemoprevention of intestinal carcinogenesis in a murine model of familial adenomatous polyposis. Nutr Cancer 60 (Suppl 1): 30-35, 2008.
40. Thompson PA, Wertheim BC, Zell JA, et al: Levels of rectal mucosal polyamines and prostaglandin E2 predict ability of DFMO and sulindac to prevent colorectal adenoma. Gastroenterology 139: 797-805, 2010.

41. Meyskens FL Jr, McLaren CE, Pelot D, et al: Difluoromethylornithine plus sulindac for the prevention of sporadic colorectal adenomas: a randomized placebo-controlled, double-blind trial. Cancer Prev Res (Phila) 1: 32-38, 2008.

42. Zell JA, Pelot D, Chen WP, McLaren CE, Gerner EW and Meyskens FL: Risk of cardiovascular events in a randomized placebo-controlled, double-blind trial of difluoromethylornithine plus sulindac for the prevention of sporadic colorectal adenomas. Cancer Prev Res (Phila) 2: 209-212, 2009.

43. Zell JA, Ziogas A, Ignatenko N, et al: Associations of a polymorphism in the ornithine decarboxylase gene with colorectal cancer survival. Clin Cancer Res 15: 6208-6216, 2009.

44. Martinez ME, O'Brien TG, Fultz KE, et al: Pronounced reduction in adenoma recurrence associated with aspirin use and a polymorphism in the ornithine decarboxylase gene. Proc Natl Acad Sci USA 100: 7859-7864, 2003.

45. Hubner RA, Muir KR, Liu JF, Logan RF, Grainge MJ and Houlston RS: Ornithine decarboxylase G316A genotype is prognostic for colorectal adenoma recurrence and predicts efficacy of aspirin chemoprevention. Clin Cancer Res 14: 2303-2309, 2008.

46. Jell J, Merali S, Hensen ML, et al: Genetically altered expression of spermidine/spermine N1-acetyltransferase affects fat metabolism in mice via acetyl-CoA. J Biol Chem 282: 8404-8413, 2007.

47. Jacobs ET, Ahnen DJ, Ashbeck EL, et al: Association between body mass index and colorectal neoplasia at follow-up colonoscopy: a pooling study. Am J Epidemiol 169: 657-666, 2009.

48. Zell JA, Lin BS, Madson N, McLaren CE, Gerner EW and Meyskens FL: Role of obesity in a randomized placebocontrolled trial of difluoromethylornithine (DFMO) + sulindac for the prevention of sporadic colorectal adenomas. Cancer Causes Control 23: 1739-1744, 2012.

49. Wallace $\mathrm{HM}$ and Fraser $\mathrm{AV}$ : Inhibitors of polyamine metabolism: review article. Amino Acids 26: 353-365, 2004.

50. Niiranen K, Pietila M, Pirttila TJ, et al: Targeted disruption of spermidine/spermine N1-acetyltransferase gene in mouse embryonic stem cells. Effects on polyamine homeostasis and sensitivity to polyamine analogues. J Biol Chem 277: 25323-25328, 2002.

51. Battaglia V, DeStefano Shields C, Murray-Stewart T and Casero RA Jr: Polyamine catabolism in carcinogenesis: potential targets for chemotherapy and chemoprevention. Amino Acids 46: 511-519, 2014.

52. Seiler N: Thirty years of polyamine-related approaches to cancer therapy. Retrospect and prospect. Part 2. Structural analogues and derivatives. Curr Drug Targets 4: 565-585, 2003.

53. Wallace HM and Niiranen K: Polyamine analogues - an update. Amino Acids 33: 261-265, 2007.

54. Belting M, Borsig L, Fuster MM, et al: Tumor attenuation by combined heparan sulfate and polyamine depletion. Proc Natl Acad Sci USA 99: 371-376, 2002.

55. Bonithon-Kopp C, Kronborg O, Giacosa A, Rath U and Faivre J: Calcium and fibre supplementation in prevention of colorectal adenoma recurrence: a randomised intervention trial. European Cancer Prevention Organisation Study Group. Lancet 356: 1300-1306, 2000.

56. Delzenne NM and Williams CM: Prebiotics and lipid metabolism. Curr Opin Lipidol 13: 61-67, 2002.

57. Food and Agriculture Organization of the United Nations and World Health Organization: Probiotics in food: health and nutritional properties and guidelines for evaluation. Food and Agriculture Organization of the United Nations, World Health Organization, Rome, 2006.

58. Boesten RJ and de Vos WM: Interactomics in the human intestine: Lactobacilli and Bifidobacteria make a difference. J Clin Gastroenterol 42 (Suppl 3): S163-S167, 2008.

59. Quigley EM: Gut bacteria in health and disease. Gastroenterol Hepatol (NY) 9: 560-569, 2013.

60. Riezzo G, Orlando A, D'Attoma B, et al: Randomised clinical trial: efficacy of Lactobacillus paracasei-enriched artichokes in the treatment of patients with functional constipation - a double-blind, controlled, crossover study. Aliment Pharmacol Ther 35: 441-450, 2012. 
61. Valerio F, de Candia S, Lonigro SL, et al: Role of the probiotic strain Lactobacillus paracasei LMGP22043 carried by artichokes in influencing faecal bacteria and biochemical parameters in human subjects. J Appl Microbiol 111: 155-164, 2011.

62. Sisto A and Lavermicocca P: Suitability of a probiotic Lactobacillus paracasei strain as a starter culture in olive fermentation and development of the innovative patented product 'probiotic table olives'. Front Microbiol 3: 174, 2012.

63. Ishikawa H, Akedo I, Otani T, et al: Randomized trial of dietary fiber and Lactobacillus casei administration for prevention of colorectal tumors. Int J Cancer 116: 762-767, 2005.

64. Zeng H, Lazarova DL and Bordonaro M: Mechanisms linking dietary fiber, gut microbiota and colon cancer prevention. World J Gastrointest Oncol 6: 41-51, 2014.

65. Kulkarni N and Reddy BS: Inhibitory effect of Bifidobacterium longum cultures on the azoxymethane-induced aberrant crypt foci formation and fecal bacterial beta-glucuronidase. Proc Soc Exp Biol Med 207: 278-283, 1994.

66. Boleij A and Tjalsma H: Gut bacteria in health and disease: a survey on the interface between intestinal microbiology and colorectal cancer. Biol Rev Camb Philos Soc 87: 701-730, 2012.

67. Singh J, Rivenson A, Tomita M, Shimamura S, Ishibashi N and Reddy BS: Bifidobacterium longum, a lactic acid-producing intestinal bacterium inhibits colon cancer and modulates the intermediate biomarkers of colon carcinogenesis. Carcinogenesis 18: 833-841, 1997.

68. Di Marzio L, Russo FP, D'Alo S, et al: Apoptotic effects of selected strains of lactic acid bacteria on a human T leukemia cell line are associated with bacterial arginine deiminase and/or sphingomyelinase activities. Nutr Cancer 40: 185-196, 2001.

69. Linsalata M, Russo F, Berloco P, et al: The influence of Lactobacillus brevis on ornithine decarboxylase activity and polyamine profiles in Helicobacter pylori-infected gastric mucosa. Helicobacter 9: 165-172, 2004.

70. Famularo G, Perluigi M, Pieluigi M, Coccia R, Mastroiacovo P and De Simone C: Microecology, bacterial vaginosis and probiotics: perspectives for bacteriotherapy. Med Hypotheses 56: 421-430, 2001

71. Orlando A, Messa C, Linsalata M, Cavallini A and Russo F. Effects of Lactobacillus rhamnosus $\mathrm{GG}$ on proliferation and polyamine metabolism in HGC-27 human gastric and DLD-1 colonic cancer cell lines. Immunopharmacol Immunotoxicol 31: 108-116, 2009.

72. Buts JP and De Keyser N: Effects of Saccharomyces boulardii on intestinal mucosa. Dig Dis Sci 51: 1485-1492, 2006.

73. Linsalata M, Russo F, Berloco P, et al: Effects of probiotic bacteria (VSL\#3) on the polyamine biosynthesis and cell proliferation of normal colonic mucosa of rats. In Vivo 19: 989-995, 2005.

74. Matsumoto M and Benno Y: Consumption of Bifidobacterium lactis LKM512 yogurt reduces gut mutagenicity by increasing gut polyamine contents in healthy adult subjects. Mutat Res 568: 147-153, 2004

75. Cederroth CR and Nef S: Soy, phytoestrogens and metabolism: A review. Mol Cell Endocrinol 304: 30-42, 2009.

76. Thompson LU, Robb P, Serraino M and Cheung F: Mammalian lignan production from various foods. Nutr Cancer 16: 43-52, 1991.

77. Albini A, Rosano C, Angelini G, et al: Exogenous hormonal regulation in breast cancer cells by phytoestrogens and endocrine disruptors. Curr Med Chem 21: 1129-1145, 2014.

78. Cassidy A: Potential risks and benefits of phytoestrogen-rich diets. Int J Vitam Nutr Res 73: 120-126, 2003.

79. Cotterchio M, Boucher BA, Manno M, Gallinger S, Okey A and Harper P: Dietary phytoestrogen intake is associated with reduced colorectal cancer risk. J Nutr 136: 3046-3053, 2006.

80. Lechner D, Kállay E and Cross HS: Phytoestrogens and colorectal cancer prevention. Vitam Horm 70: 169-198, 2005

81. Hawrylewicz EJ, Zapata JJ and Blair WH: Soy and experimental cancer: animal studies. J Nutr 125: 698S-708S, 1995.

82. Linsalata M, Messa C, Russo F, Cavallini A and Di Leo A: Estrogen receptors and polyamine levels in human gastric carcinoma. Scand J Gastroenterol 29: 67-70, 1994

83. Russo F, Linsalata M, Messa C, et al: Polyamines and estrogen-receptor concentrations in human colorectal carcinomas. Ital J Gastroenterol 24: 8-12, 1992.

84. Cross HS, Kallay E, Lechner D, Gerdenitsch W, Adlercreutz H and Armbrecht HJ: Phytoestrogens and vitamin D metabolism: a new concept for the prevention and therapy of colorectal, prostate, and mammary carcinomas. J Nutr 134: 1207S-1212S, 2004.
85. Booth C, Hargreaves DF, Hadfield JA, McGown AT and Potten CS: Isoflavones inhibit intestinal epithelial cell proliferation and induce apoptosis in vitro. Br J Cancer 80: 1550-1557, 1999.

86. Li HQ, Luo Y and Qiao CH: The mechanisms of anticancer agents by genistein and synthetic derivatives of isoflavone. Mini Rev Med Chem 12: 350-362, 2012.

87. Shon YH, Park SD and Nam KS: Effective chemopreventive activity of genistein against human breast cancer cells. J Biochem Mol Biol 39: 448-451, 2006.

88. Linsalata M, Russo F, Notarnicola M, et al: Effects of genistein on the polyamine metabolism and cell growth in DLD-1 human colon cancer cells. Nutr Cancer 52: 84-93, 2005.

89. Tanaka T, Makita H, Kawabata K, et al: Chemoprevention of azoxymethane-induced rat colon carcinogenesis by the naturally occurring flavonoids, diosmin and hesperidin. Carcinogenesis 18: 957-965, 1997

90. Au A, Li B, Wang W, Roy H, Koehler K and Birt D: Effect of dietary apigenin on colonic ornithine decarboxylase activity, aberrant crypt foci formation, and tumorigenesis in different experimental models. Nutr Cancer 54: 243-251, 2006.

91. Linsalata M, Orlando A, Messa C, Refolo MG and Russo F: Quercetin inhibits human DLD-1 colon cancer cell growth and polyamine biosynthesis. Anticancer Res 30: 3501-3507, 2010.

92. Gosse F, Roussi S, Guyot S, et al: Potentiation of apple procyanidin-triggered apoptosis by the polyamine oxidase inactivator MDL 72527 in human colon cancer-derived metastatic cells. Int J Oncol 29: 423-428, 2006

93. Ibanez C, Simo C, Garcia-Canas V, Gomez-Martinez A, Ferragut JA and Cifuentes A: CE/LC-MS multiplatform for broad metabolomic analysis of dietary polyphenols effect on colon cancer cells proliferation. Electrophoresis 33: 2328-2336, 2012.

94. Wallerath T, Deckert G, Ternes T, et al: Resveratrol, a polyphenolic phytoalexin present in red wine, enhances expression and activity of endothelial nitric oxide synthase. Circulation 106: 1652-1658, 2002.

95. Sengottuvelan M, Senthilkumar R and Nalini N: Modulatory influence of dietary resveratrol during different phases of 1,2-dimethylhydrazine induced mucosal lipid-peroxidation, antioxidant status and aberrant crypt foci development in rat colon carcinogenesis. Biochim Biophys Acta 1760: 1175-1183, 2006.

96. Wolter F and Stein J: Resveratrol enhances the differentiation induced by butyrate in caco- 2 colon cancer cells. J Nutr 132 : 2082-2086, 2002.

97. Schneider Y, Vincent F, Duranton B, et al: Anti-proliferative effect of resveratrol, a natural component of grapes and wine, on human colonic cancer cells. Cancer Lett 158: 85-91, 2000.

98. Schneider Y, Duranton B, Gosse F, Schleiffer R, Seiler N and Raul F: Resveratrol inhibits intestinal tumorigenesis and modulates host-defense-related gene expression in an animal model of human familial adenomatous polyposis. Nutr Cancer 39: 102-107, 2001.

99. Wolter F, Turchanowa L and Stein J: Resveratrol-induced modification of polyamine metabolism is accompanied by induction of c-Fos. Carcinogenesis 24: 469-474, 2003.

100. Ulrich S, Loitsch SM, Rau O, et al: Peroxisome proliferator-activated receptor gamma as a molecular target of resveratrol-induced modulation of polyamine metabolism. Cancer Res 66: 7348-7354, 2006.

101. Wolter F, Ulrich S and Stein J: Molecular mechanisms of the chemopreventive effects of resveratrol and its analogs in colorectal cancer: key role of polyamines? J Nutr 134: 3219-3222, 2004.

102. Henning SM, Wang P, Abgaryan N, et al: Phenolic acid concentrations in plasma and urine from men consuming green or black tea and potential chemopreventive properties for colon cancer. Mol Nutr Food Res 57: 483-493, 2013.

103. Yang CS, Li G, Yang Z, Guan F, Chen A and Ju J: Cancer prevention by tocopherols and tea polyphenols. Cancer Lett: Feb 8, 2013 (Epub ahead of print).

104. Kumazaki M, Noguchi S, Yasui Y, et al: Anti-cancer effects of naturally occurring compounds through modulation of signal transduction and miRNA expression in human colon cancer cells. J Nutr Biochem 24: 1849-1858, 2013.

105. Melgarejo E, Urdiales JL, Sanchez-Jimenez F and Medina MA: Targeting polyamines and biogenic amines by green tea epigallocatechin-3-gallate. Amino Acids 38: 519-523, 2010. 
106. Bachrach U and Wang YC: Cancer therapy and prevention by green tea: role of ornithine decarboxylase. Amino Acids 22: $1-13,2002$.

107.Paul B, Hayes CS, Kim A, Athar M and Gilmour SK: Elevated polyamines lead to selective induction of apoptosis and inhibition of tumorigenesis by (-)-epigallocatechin-3-gallate (EGCG) in ODC/Ras transgenic mice. Carcinogenesis 26: 119-124, 2005.

108. Chung JY, Huang C, Meng X, Dong Z and Yang CS: Inhibition of activator protein 1 activity and cell growth by purified green tea and black tea polyphenols in H-ras-transformed cells: structure-activity relationship and mechanisms involved. Cancer Res 59: 4610-4617, 1999.

109.Gupta S, Ahmad N, Marengo SR, MacLennan GT, Greenberg NM and Mukhtar H: Chemoprevention of prostate carcinogenesis by alpha-difluoromethylornithine in TRAMP mice. Cancer Res 60: 5125-5133, 2000.

110. Milovic V, Turchanowa L, Stein J and Caspary WF: Transepithelial transport of putrescine across monolayers of the human intestinal epithelial cell line, Caco-2. World J Gastroenterol 7: 193-197, 2001.

111. Zoumas-Morse C, Rock CL, Quintana EL, Neuhouser ML, Gerner EW and Meyskens FL Jr: Development of a polyamine database for assessing dietary intake. J Am Diet Assoc 107: 1024-1027, 2007.

112. Loser C, Eisel A, Harms D and Folsch UR: Dietary polyamines are essential luminal growth factors for small intestinal and colonic mucosal growth and development. Gut 44: 12-16, 1999.

113. Seiler N, Sarhan S, Grauffel C, Jones R, Knodgen B and Moulinoux JP: Endogenous and exogenous polyamines in support of tumor growth. Cancer Res 50: 5077-5083, 1990.

114. Muth A, Madan M, Archer JJ, Ocampo N, Rodriguez L and Phanstiel O: Polyamine transport inhibitors: design, synthesis, and combination therapies with difluoromethylornithine. J Med Chem 57: 348-363, 2014.
115. Ignatenko NA, Besselsen DG, Roy UK, et al: Dietary putrescine reduces the intestinal anticarcinogenic activity of sulindac in a murine model of familial adenomatous polyposis. Nutr Cancer 56: 172-181, 2006.

116. Yerushalmi HF, Besselsen DG, Ignatenko NA, et al: Role of polyamines in arginine-dependent colon carcinogenesis in Apc(Min) (/+) mice. Mol Carcinog 45: 764-773, 2006.

117. Gerner EW: Impact of dietary amino acids and polyamines on intestinal carcinogenesis and chemoprevention in mouse models. Biochem Soc Trans 35: 322-325, 2007.

118. Ignatenko NA, Gerner EW and Besselsen DG: Defining the role of polyamines in colon carcinogenesis using mouse models. J Carcinog 10: 10, 2011.

119. Zell JA, Ignatenko NA, Yerushalmi HF, et al: Risk and risk reduction involving arginine intake and meat consumption in colorectal tumorigenesis and survival. Int J Cancer 120: 459-468, 2007.

120. Vargas AJ, Wertheim BC, Gerner EW, Thomson CA, Rock CL and Thompson PA: Dietary polyamine intake and risk of colorectal adenomatous polyps. Am J Clin Nutr 96: 133-141, 2012.

121. Raj KP, Zell JA, Rock CL, et al: Role of dietary polyamines in a phase III clinical trial of difluoromethylornithine (DFMO) and sulindac for prevention of sporadic colorectal adenomas. Br J Cancer 108: 512-518, 2013.

122.Cipolla BG, Havouis R and Moulinoux JP: Polyamine reduced diet (PRD) nutrition therapy in hormone refractory prostate cancer patients. Biomed Pharmacother 64: 363-368, 2010 . 